\title{
SUPLEMENTACIÓN NUTRICIONAL DE VERANERA (CRATYLIA ARGENTEA) Y CAÑA DE AZÚCAR (SACCHARUM OFFICINARUM) A VACAS PRODUCTORAS DE LECHE
}

\author{
Yader Ramón Morales Lara ${ }^{[22]}$ \\ Junier Samuel Herrera Maradiaga ${ }^{[23]}$
}

\section{Resumen}

En el estudio se realizó un análisis de las bondades nutricionales de la ración integrada por la caña de azúcar (Saccharum officinarum) y Veranera (Cratylia argentea) como fuentes energética y proteica respectivamente, en la producción y calidad de la leche, analizando la viabilidad económica de la misma. Esto se hizo con el propósito de formular recomendaciones que mejoren el manejo de la lechería y contribuyan a la eficiencia productiva del hato bovino lechero.

El experimento se realizó durante las dos épocas del año ${ }^{[24]}$, analizando en cada una de las épocas, un período antes, durante y después de la suplementación, se suministraron $20 \mathrm{Kg} /$ vaca/día de la ración ${ }^{[25]}$ a diez vacas en producción láctea seleccionadas bajos parámetros ${ }^{[26]}$ definidos con anterioridad.

En cuanto a la producción láctea se registró un diferencia de $1.43 \mathrm{Kg} / \mathrm{vaca} / \mathrm{día}$ en la época seca y $0.83 \mathrm{Kg} / \mathrm{vaca} /$ día para la época lluviosa con respecto a las vacas testigos manejadas de manera tradicional, de manera similar ocurrió con los porcentajes de grasa y proteína obteniendo incrementos de y $0.49 \%$ y $0.14 \%$ respectivamente, esto añadido a que se ha identificó una rentabilidad del $350 \%$, y que la leguminosa Cratylia argentea presenta rendimiento de materia verde y seca para la Cratylia argentea en las dos épocas del año de 60.8 ton/Mv/ha/ año y $13.92 \mathrm{ton} / \mathrm{ms} / \mathrm{ha} /$ año. Se hace necesario promover aún más esta tecnología en busca de mejorar el nivel de vida de las familias productoras, además de reducir el impacto ambiental generado por la ganadería extensiva.

[22] Ing. Zootecnista, URACCAN, Recinto Nueva Guinea, yader77@yahoo.com

[23] Ing. Zootecnista, BANEX Nueva Guinea.

[24] Seca y lluviosas treinta días para cada época.

[25] $25 \%$ de Veranera y $75 \%$ caña de azúcar).

[26] Número de partos, edad, período de lactancia, estado corporal. 


\section{SISTEMA AGROFORESTAL Y PRODUCCIÓN PECUARIA}

\section{Introducción}

Nicaragua es un país eminentemente agropecuario y la producción pecuaria en los últimos 10 años ha tenido un gran repunte en el Producto Interno Bruto (PIB) fundamentalmente las exportaciones de carne y leche (CENAGRO, 2004).

Uno de los factores que más afectan la producción ganadera es el déficit de proteína y otros principios nutritivos en los pastizales, lo que afecta los rendimientos y calidad de la producción de leche específicamente la grasa y la proteína, además de los indicadores reproductivos, influyendo directamente en la economía pecuaria (PRA-DC/IDR, 2005).

Recientemente se ha introducido a nuestro municipio una especie de leguminosa, veranera (Cratylia argentea) caracterizada por ser un arbusto que se ramifica a partir de la base del tallo alcanzando hasta 3 metros de altura que presenta buenos rendimientos de producción de biomasa y buena capacidad de rebrote tanto en época seca como en época lluviosa según estudios realizados en nuestra zona (Miranda, 2004).

Basados en lo expuesto y dado que aún no se han realizado estudios para determinar las bondades nutricionales de esta especie, es que se propuso realizar la investigación con la especie antes mencionada complementada con la caña de azúcar (Saccharum officinarum), con el fin de determinar el efecto sobre la producción y la calidad de la leche, utilizando recursos baratos y sostenibles que no compiten con la alimentación humana e incidan en la economía pecuaria.

\section{Revisión de literatura}

\section{Bancos forrajeros}

Los bancos forrajeros son áreas en las cuales los árboles y los arbustos forrajeros se cultivan en bloques compactos y a alta densidad, con miras a aprovechar el terreno y aumentar la producción de forrajes de alta calidad nutritiva. Para que un sistema se pueda llamar banco de proteína, el forraje que produce debe contener más del $15 \%$ de proteína cruda. Para esto deben establecerse especies leguminosas que además de contener alto grado de proteína produzcan grandes cantidades de forraje. (Pizarro (1995). 
Estos pueden ser cosechados y llevados a los animales en un sistema de corte $\mathrm{y}$ acarreo o pueden ser pastoreados directamente en periodos cortos de una hora y media a dos horas por día (Ibíd.).

Tomando en cuenta éstas definiciones y en vista que, veranera (Cratylia argéntea) es una especie que está presente en el municipio desde el año 2004 y ha sido objeto de investigaciones para medir su capacidad productiva bajo diferentes densidades de siembra comprobando así su capacidad para adaptarse al clima y suelos de la zona, de acuerdo a resultados obtenidos en estudios realizados por Miranda (2004).

Por lo anterior y atendiendo recomendaciones de investigadores anteriores, se propuso realizar esta investigación con la finalidad de identificar el efecto que pueda ejercer esta especie en la producción y calidad de la leche de bovinos, de tal manera que pueda transferirse esta tecnología a productores de la zona y transformen su sistema de producción tradicional por otro más rentable.

\section{Características de especie utilizadas como suplemento alimenticio}

\section{Origen y descripción botánica de veranera (Cratylia argentea)}

El género Cratylia pertenece a la familia leguminosae, subfamilia, Papilionydae, esta es nativa de la Amazonia central de Brasil y de áreas de Perú, Bolivia y del noreste de Argentina, en Brasil crece de forma natural (Pizarro 1995).

\section{Adaptación a climas y suelos}

Cratylia argentea se adapta bien a un amplio rango de sitios localizados entre o y 900 msnm. La planta crece bien en suelos bien drenados de buena y moderada fertilidad tipo inseptisol, y en ecosistemas sub-Húmedos con 5 a 6 meses secos; aunque también crece bien en utisoles de baja fertilidad. Según Pizarro (1995) la Cratylia argentea no crece bien en suelos calcáreos ni en suelos pesados con tendencia a saturarse con humedad; en estos últimos es común observar alta mortalidad de plantas durante la fase de establecimiento.

Las plántulas adultas de este cultivar toleran el fuego y pueden rebrotar de yemas presente en la corona de la raíz, una de las característica más importantes es su capacidad de rebrote y retención de hojas durante la época seca, lo que está asociado con el desarrollo de raíces vigorosas que alcanzan hasta 2 metros de longitud, Pizarro (1995). 


\section{SISTEMA AGROFORESTAL Y PRODUCCIÓN PECUARIA}

\section{Establecimiento}

Veranera (Cratylia argéntea) se propaga fácilmente por semilla; por el contrario, la propagación vegetativa por estaca no ha sido exitosa (Pizarro et al., 1995) la semilla debe ser colocada de manera superficial (menos de $2 \mathrm{~cm}$. de profundidad en el suelo) ya que a mayor profundidad el surgimiento es muy bajo y el desarrollo de la plántula es muy lento, la semilla no requiere de escarificación antes de la siembra debido a la poca dureza que presenta (CIAT, 1991).

La siembra directa en el campo bajo condiciones de labranza mínima o después de una labranza convencional con arado y pase de rastra ha dado buenos resultados; también se puede hacer un almácigo en bolsas y posteriormente las plántulas desarrolladas se llevan al campo. Este último sistema ofrece un establecimiento más seguro, pero resulta más costoso que el sistema de siembra directa; además, requiere condiciones ambientales favorables y plántulas con un mínimo de tres a cuatro hojas al momento del trasplante ((ibíd.).

El crecimiento de las plantas de veranera (Cratylia argéntea) es lento durante los primeros 2 meses después de la siembra, a pesar de que el vigor de las plántulas es mayor que de otras leguminosas arbustivas como leucaena (Leucaena leucocephala) (Ibíd.).

\section{Densidad de siembra y frecuencia de corte}

Según Miranda (2004) en estudios realizados en el municipio de Nueva Guinea, se determinó que la densidad de siembra con el mayor rendimiento de materia fresca total fue en 40,000 plantas por ha $(0.50 \times 0.50)$ obteniéndose un rendimiento promedio de 67.15 ton /ha /año, que difiere de las densidades de 20,000 plantas ha (0.5 x $1 \mathrm{~m}$. entre plantas) con un rendimiento de 41.98 ton / ha/añoy la densidad de 10,000 plantas/ha (1 $\times 1 \mathrm{~m}$. entre plantas) con un rendimiento de 27.16 ton de ha/ año

\section{Primer corte}

Rebrotar con vigor después de un corte requiere que los arbustos y árboles hayan desarrollado suficientemente su sistema radicular para soportar el rebrote. Por lo tanto, realizar el primer corte demasiado temprano (en un estado inmaduro) o demasiado tarde (en un estado senescente) puede reducir el vigor del rebrote significativamente (Maass, et al., s.f.).

Los rendimientos foliares y leñosos de varias especies durante el año posterior al primer corte (entre 13 y 21 meses después de la siembra) aumentaron a medida 
que la edad al primer corte fue mayor (Eila, \& et al., 1991; Stür, et al., 1994 citados por Maass, et al., s.f.).

En la mayoría de los trabajos revisados Maass, (1995), las alturas de corte más altas se relacionaron con altos rendimientos de MS, estando la altura de corte óptima entre 90 y $120 \mathrm{~cm}$. No obstante, estos mismos autores reportaron que en algunos casos la altura de corte no afectó el rendimiento de MS. Por ejemplo, no encontraron diferencias en producción de MS de veranera (Cratylia argéntea) cuando se cosechó a 20 ó $40 \mathrm{~cm}$.

\section{Calidad Nutritiva de veranera (Cratylia argentea)}

Resultados de análisis químicos realizados en muestras de leguminosas arbustivas cosechadas en la estación CIAT Quilichao, mostraron que el follaje comestible (hojas + tallos finos) de Cratylia argentea (3 meses de rebrote) tuvo un contenido de proteína cruda (23.5\%) similar al de otras especies conocidas como Calliandra calothyrsus (23.9\%), Erythrina popegiana (27.1\%), Gliricidia sepium (25.45) y Leucaena leucocephala (26.5\%) (Perdomo, 1991 citado por Argel y Lascano, 1995).

Las leguminosas tienen la cualidad de mantener su calidad a través del tiempo, especialmente su contenido de proteína cruda durante la época seca que es cuando más la consumen los animales. Por el contrario, las gramíneas tropicales presentan una disminución acelerada de su calidad nutritiva al avanzar la edad de la planta. Durante la época seca o cuando el suministro de nitrógeno es deficiente, las gramíneas presentan contenidos de proteína cruda inferiores al 7\% (Lascano, 1995) en la materia seca, lo cual se traduce en una disminución del consumo voluntario y consecuentemente de la producción animal.

Como resultado del su alto contenido de proteína cruda y bajos niveles de taninos. Cratylia argentea es una excelente fuente de nitrógeno fermentable en el rumen (Ibíd.).

\section{Respuesta en vacas de ordeño al suministro de Cratylia argentea}

Trabajos experimentales realizados con vacas en ordeño de los tipos Holstein y Cebú, pastoreando Brachiaria decumbens (pasto peludo) y suplementadas con caña de azúcar y niveles crecientes de Cratylia argentea mostraron que, estas últimas no incrementaron significativamente la producción de leche al aumentar el nivel de Cratylia argentea de $25 \%$ a $75 \%$ en la dieta, mientras que las vacas tipo Hosltein aumentaron su producción un $25 \%$ (1.6 kg/vaca/día) cuando se ofreció un suplemento combinado de 25 \% de caña de azúcar y 75\% de Cratylia argentea (CIAT1997). 


\section{SISTEMA AGROFORESTAL Y PRODUCCIÓN PECUARIA}

Según Lascano 1,995 en diferentes niveles de Suplementación de veranera (Cratylia argéntea) y caña de azúcar (Saccharum officinarum) a vacas lecheras en pastoreo los resultados mostraron que la suplementación resulto en aumentos crecientes de producción de leche (1.2 a 2.2 lts/vaca/día) a medida que se incrementó la proporción de veranera (Cratylia argéntea).

\section{Caña de azúcar (Saccharum officinarum)}

Las plantas de hábitos perennes, crecen en matojos robustos, los tallos son jugosos y alcanzan una alturas de 2 a 3 metros y diámetros de 2 a $6 \mathrm{~cm}$. Poseen entrenudos y vainas envolventes que se desprenden de los tallos, las hojas son alargadas, de $4 \mathrm{~cm}$ a $6 \mathrm{~cm}$ de anchas.

La caña de azúcar (Saccharum officinarum) se adapta bien a suelos franco arcillosos con un $\mathrm{PH}$ entre 5,5 a 7,5, se propaga mediante estacas o tallos con un buen número de yemas viables que se colocan en los surcos y se cubren con una capa de 1,5 a 2,5 $\mathrm{cm}$. del suelo, la siembra debe hacerse al inicio de la época lluviosa, a menos que se cuente con riego (Urdaneta, 2005)

Manejo: la caña de azúcar (Saccharum officinarum) debe cosecharse haciendo cortes cada 10 a 12 semanas, así se puede efectuar hasta 4 cortes por año, en condiciones naturales. Con riego se puede efectuar hasta 6 cortes por año (Ibíd.).

La caña de azúcar (Saccharum officinarum) es una gramínea que proporciona un elevado rendimiento de forraje por área, alcanzando promedios de 200 t/há/año de forraje verde (tallo+hojas) y hasta 450 t/há/año en Colombia, cortada a intervalos de 10-12 meses (Ibíd.).

Estas producciones de caña de azúcar (Saccharum officinarum) como forraje, equivalen a mantener en época seca entre 36-88 animales por hectárea, cuando las producciones de forraje verde de caña (hoja + tallo) se encuentran entre 65 y $158 \mathrm{tn} /$ ha necesitándose $1,8 \mathrm{t}$ de caña de azúcar repicada por animal (de $450 \mathrm{Kg}$ PV) durante 90 días (Urdaneta, 2005).

\section{Calidad del forraje}

La característica más relevante es su alto contenido de azúcares solubles combinado con una fibra altamente lignificada, originando una baja digestibilidad de la fibra en el animal, esto unido a un bajo contenido de proteínas y minerales y a una ausencia casi total de grasas y almidones hace que no se recomiende su utilización como única fuente de alimento en la alimentación del ganado doble propósito (Urdaneta, 2005). 
Cuando se utilice la caña de azúcar (Saccharum officinarum) como un alimento complementario al pasto (pastoreo o escasez de pasto), se debe agregar proteína para mejorar su valor nutricional, la cual puede ser en forma de urea disuelta en agua y mezclada con la caña integral repicada o utilizar fuentes alternativas en el follaje de leguminosas como (Leucaena leucocephala) y madero negro (Gliricidia sepium), así como la hoja de la yuca (Manihot esculenta) y del quinchoncho (Cajanus cajan) entre otras (Ibíd.).

Valor nutricional de la Caña de azúcar (Saccharum officinarum) y algunos pastos

\begin{tabular}{lllll}
\hline Indicadores & Caña de azúcar & Pasto Elefante & King grass & $\begin{array}{c}\text { Pastos en } \\
\text { general }\end{array}$ \\
\hline MS\% & $26,2-28,7$ & $19,5-25$ & $17-20,4$ & $22-33,7$ \\
\hline Proteína \% & $2,6-4,7$ & $7,2-9,85$ & $9-8,8$ & $6-10$ \\
\hline Fibra \% & $36,1-48,1$ & $28,6-36,1$ & $31,9-32,2$ & $28-41,9$ \\
\hline Digestibilidad \% & $50-60$ & $60,8-71,7$ & $62,7-71,9$ & $40-55$ \\
\hline
\end{tabular}

Fuente: Urdaneta (2005).

Cosecha de la caña de azúcar (Saccharum officinarum) como alimento. La cantidad de caña de azúcar a cosechar está en función del número de animales que se tiene que alimentar, y sí se conoce el peso promedio de la caña, el corte se puede programar por número de cañas, longitud del surco o cantidad de surcos se puede programar 1 ó 2 veces por semana. La caña después de cortada se almacena bajo sombra y puede durar hasta un mes sin perder su valor nutricional a nivel del tallo (Urdaneta, 2005).

Se puede alimentar hasta 8 unidades animales con plantíos bien establecidos y manejados durante los 5 meses de verano o 18 animales durante 2 meses.

\section{Nutrición de vacas lecheras}

La suplementación con $C$. argentea, es que esta leguminosa contribuye a aliviar las deficiencias de proteína de rumiantes que son comunes en la época seca dada la alta degrababilidad de su proteína en el rumen. Por otra parte, el efecto positivo de $C$. argentea como suplemento en sistemas de corte y acarreo sería mayor si se combina con una fuente rica en energía como la caña de azúcar. (Lascano, 2003)

En base en lo anterior se diseñaron una serie de ensayos en el CIAT en los cuales se suplementó (1.5\% de MS del PV) diferentes niveles de C. argentea y caña de azúcar a vacas lechera en pastoreo. Los resultados (Avila y Lascano, no publicados) mostraron que la suplementación resultó en aumentos crecientes de producción de leche (1.2 a 


\section{SISTEMA AGROFORESTAL Y PRODUCCIÓN PECUARIA}

2.2 litros por vaca/d) a medida que se incrementó la proporción de C. argentea (0, 25, 50 y $75 \%$ ) en el suplemento. Sin embargo, la respuesta a la inclusión de C. argentea en el suplemento dependió del potencial de producción de leche de las vacas y de la calidad de la gramínea en la pastura. Vacas con poco potencial de producción de leche (3-4 litros) no respondieron a la suplementación con C. argentea. Tampoco se observó respuesta a la suplementación caña/C. argentea cuando la gramínea (hojas) en la pastura utilizada por las vacas tenia niveles de proteína de más de 7\%. (Lascano, 2003)

\section{Ingestión de Materia Seca (IMS)}

Inducir a que la vaca coma grandes cantidades de alimentos, es la clave para obtener una producción de leche eficiente y productiva. Haga una buena selección de los ingredientes, para asegurar una ingestión máxima. Todos los alimentos que la vaca requiere para la producción de leche (excepto el agua), se encuentran en la materia seca de los alimentos. Una alta ingestión de materia seca (IMS) da como resultado a una ingestión alta de nutrientes y un rendimiento alto de la producción de leche. (pagina de informacion ganadera de ray del pino, 2000)

La tabla 1 muestra los máximos niveles de IMS (de forrajes y mezclas de cereales) que las vacas de ordeño pueden comer, durante los períodos de la mitad y el final de la lactación. La tabla da los valores de IMS, en \% de su peso corporal y en Kg por día. Una vaca que pesa $550 \mathrm{~kg}$, ordeñando $30 \mathrm{~kg}$ de leche/día, puede comer un 3.7\% de su cuerpo corporal de MS (materia seca), que representa unos $20.4 \mathrm{~kg} /$ día. Una vaca más grande de $(650 \mathrm{~kg}$ ) y con la misma producción de leche/día, puede comer únicamente el $3.4 \%$ de su peso corporal en MS, unos (22.1 kg por día). Vacas más grandes, con una mayor producción de leche/día, pueden comer más cantidad de MS. (Ibid) Ingestión de Materia Seca (IMS) en Kg/Día y en \% del peso corporal durante los períodos de la mitad y final de lactación.

\begin{tabular}{llll}
\hline $\begin{array}{c}\text { Producción } \\
\text { diaria }\end{array}$ & $\begin{array}{c}\text { Peso corporal de la } \\
\text { Vaca 450 }(\mathrm{Kg})\end{array}$ & $\begin{array}{c}\text { Peso corporal de la } \\
\text { Vaca 550(Kg) }\end{array}$ & $\begin{array}{l}\text { Peso corporal de } \\
\text { la Vaca 650(Kg) }\end{array}$ \\
\hline $\begin{array}{l}\text { Kgs de Leche/ } \\
\text { Día }\end{array}$ & $\begin{array}{l}\text { (\% del peso) }(\mathrm{IMS} / \mathrm{Kg} / \\
\text { día) }\end{array}$ & $\begin{array}{l}\text { (\% del peso) }(\mathrm{IMS} / \mathrm{Kg} / \\
\text { día })\end{array}$ & $\begin{array}{l}\text { (\% del peso) (IMS/ } \\
\mathrm{Kg} / \text { día) }\end{array}$ \\
\hline 10 & 2.611 .7 & 2.312 .7 & 2.112 .0 \\
\hline 20 & 3.415 .3 & 3.016 .5 & 2.818 .2 \\
\hline 30 & 4.218 .9 & 3.720 .4 & 3.422 .1 \\
\hline 40 & 5.022 .5 & 4.323 .7 & 3.824 .7 \\
\hline
\end{tabular}




\begin{tabular}{llll}
\hline $\begin{array}{c}\text { Producción } \\
\text { diaria }\end{array}$ & $\begin{array}{c}\text { Peso corporal de la } \\
\text { Vaca 450 }(\mathbf{K g})\end{array}$ & $\begin{array}{c}\text { Peso corporal de la } \\
\text { Vaca 550 }(\mathbf{K g})\end{array}$ & $\begin{array}{c}\text { Peso corporal de } \\
\text { la Vaca 650(Kg) }\end{array}$ \\
\hline 50 & 5.625 .2 & 5.027 .5 & 4.428 .6 \\
\hline
\end{tabular}

(pagina de informacion ganadera de ray del pino, 2000)

\section{Características generales de la Leche}

\section{¿Qué es la leche?}

Es aquel alimento producido por las hembras mamíferas después del parto y su objetivo es alimentar sus crías en la primera fase de la vida. Con la intervención del ser humano se ha desarrollado la producción láctea de determinadas hembras domésticas, principalmente las vacas, para obtener un excedente considerable y utilizarlo en la alimentación humana, ya sea como leche fresca o como producto transformado. (Fundación Hogares Juveniles Campesinos , 2002).

\section{Composición de la leche de diferentes especies}

\begin{tabular}{llll}
\hline \multirow{2}{*}{ Características } & \multicolumn{3}{c}{ Especies } \\
\cline { 2 - 4 } & \multicolumn{1}{c}{ vaca } & oveja & cabra \\
\hline Calorías & 68,0 & 104,0 & 75,0 \\
\hline Proteína & 3.3 & 5,5 & 3,8 \\
\hline grasa & 3.6 & 7,0 & 4,3 \\
\hline Carbohidratos & 4.8 & 4,3 & 4,6 \\
\hline agua & 87,0 & 82,4 & 86,3 \\
\hline
\end{tabular}

(Fundación Hogares Juveniles Campesinos , 2002)

\section{Materiales y métodos}

\section{Ubicación del estudio}

El experimento se realizó en la finca "Rancho Bonanza" propiedad del productor Roberto Mora Valdez, ubicada en la Colonia el Níspero a $19 \mathrm{Km}$. de la ciudad de Nueva Guinea carretera a Managua en la Región Autónoma del Atlántico Sur (RAAS), la finca cuenta con una extensión de 600 manzanas, donde el 100 \% del área está destinada 
a la producción pecuaria y el propósito principal es la producción de leche, la mayor parte está ocupada con pastos naturales y en menor proporción pastos mejorados.

\section{Tipo de estudio}

\section{El estudio se clasifica de la siguiente forma:}

Según el período como longitudinal ya que la investigación se realizó durante la época seca y lluviosa con las mismas vacas en estudio con lo que se analizó todo el período de lactancia de las 10 vacas.

Según el análisis y alcance de los resultados como experimental - descriptivo ya que se experimentó el comportamiento de la ración de veranera (Cratylia argéntea) y caña de azúcar (saccharum officinarum) como suplemento alimenticio y sus efectos sobre la producción y calidad de la leche y se describe cada uno de los resultados.

\section{Población y Muestra}

La población a evaluar es el ganado bovino en producción de leche en la época seca y lluviosa constituida por 70 vacas paridas.

La muestra evaluada fue de 10 vacas en producción láctea que representa el $14.2 \%$ de la población, tanto para la época seca como lluviosa del año 2006.

Los criterios para seleccionar la muestra fueron: el encaste, número de partos, edad, periodo de lactancia, condición física.

\section{Tipo de Muestreo}

El tipo de muestreo fue no probabilístico por conveniencia debido a que seleccionamos la muestra de acuerdo a algunas características descritas anteriormente.

\section{Descripción del Estudio}

El estudio consistió en evaluar la producción láctea a través de la suplementación de veranera (Cratylia argéntea) y caña de azúcar (Saccharum oficcinarum) como fuente de proteínas y energía respectivamente, cabe destacar que el área de cultivo para ambas especies ya había sido establecida, con un área de 1.25 manzanas de veranera (Cratylia argéntea) y 3 manzanas de caña de azúcar (Saccharum oficcinarum). 
Este estudio se efectuó en las épocas seca y lluviosa del año 2006 siguiendo un cronograma de actividades previamente establecido. El estudio se llevó a cabo por un periodo de 60 días, lo que se distribuyó 30 días para cada época evaluada. Cada época en estudio estuvo distribuida en 3 etapas de 10 días cada una. (Antes, durante y después de la suplementación) en cada una de las etapas se midió la producción láctea y se realizó análisis de laboratorio en el equipo de análisis ultrasónico de leche denominado EKOMILK para lo cual se tomó muestra de leche por cada vaca suplementada y de vacas testigo. Los análisis fueron realizados en el laboratorio de la unidad de mejoramiento genético presente en la universidad URACCAN.

\section{Tipo de ensayo}

Aunque no se haya establecido un diseño experimental como tal por el hecho de definir el muestreo como no probabilístico por conveniencia dadas las características del estudio explicados con anterioridad. El ensayo fue manejado mediante un diseño experimental de tipo DCA (Diseño Completamente al Azar).

\section{Tratamientos}

T 1: Formado por 5 vacas en producción de leche, suplementadas cada una con el $25 \%$ (5 kg) de veranera (Cratylia argéntea) más 75\% (15 kg) de caña de azúcar (Saccharum officinarum).

La alimentación se preparó diariamente y se proporcionó en comederos ubicados en el área de ordeño, las vacas permanecieron estabuladas después de finalizar el ordeño hasta el medio día, posteriormente se incorporaron al pastoreo. Para determinar la ración brindada se tomó en cuenta el peso vivo tomando como promedio $400 \mathrm{~kg}$ por vaca sabiendo que un bovino consume el $10 \%$ de su peso vivo lo que equivale a 40 kgmv/dia y se suplementó el 50 \% del consumo diario.

T 2: consta de 5 vacas, a las que se les manejó de manera tradicional (pastoreo) en pastizales naturales y mejorados, éstas eran incorporadas al pastoreo después del ordeño se debe aclarar que en las 5 unidades de análisis de cada tratamiento hubo representación de los tres tipos de encaste incluidos: 2 Holstein, 2 Pardo Suizo, 1 Reina por tratamiento. 


\section{SISTEMA AGROFORESTAL Y PRODUCCIÓN PECUARIA}

\section{Variables a Medir}

\section{Efecto de la veranera (Cratylia argéntea) y Caña de Azúcar (Saccharum Oficcinarum) sobre la producción y calidad de la leche}

Este se midió a través de los resultados obtenidos en la producción, realizando pesajes diarios de la leche de las 10 vacas (5 suplementadas y 5 sin suplemento) por un periodo de 30 días en cada época evaluada.

Con respecto a la calidad de la leche se tomó una muestra de leche por cada vaca en estudio ( 5 con suplemento y 5 sin suplemento) y se le hizo análisis en laboratorio por cada muestra tomada mediante equipo de análisis ultrasónico de leche denominado EKOMILK, producto de este procedimiento y basados en los estándares de calidad de la leche (\% grasa y proteína) interpretamos los resultados realizando un total de 60 muestras correspondiente a ambos períodos de evaluación.

\section{Producción de MV y MS de veranera (Cratylia argéntea) y Caña de azúcar (Saccharum Oficcinarum)}

Para la medición de materia verde (MV) y materia seca (MS) se utilizó el sistema de recolección con el metro cuadrado para la toma de las muestras, cortando las plantas a una altura de $25 \mathrm{~cm}$. del suelo, se pesó y el resultado de este se multiplicó por el área total utilizada y se obtuvo los rendimientos por área. Para medir la producción de materia verde y materia seca de veranera (Cratylia argéntea) de la población de 10,000 plantas establecidas a una distancia entre planta y planta de $1 \mathrm{x} 1 \mathrm{~m}$ de Cratylia argentea, se tomaron en cuenta tres muestras de un metro cuadrado.

El material utilizado para obtener la materia verde (plantas) se sometió a deshidratación en un horno, bajo supervisión y control de la temperatura, se pesó diariamente para ver los avances y cuando se obtuvo el mismo peso por tres días consecutivos se determino el peso final o materia seca (MS) de la veranera (Cratylia argéntea).

El procedimiento anterior se realizó para determinar la materia verde y seca de la veranera (Cratylia argéntea) como también de la caña de azúcar (sackcharum Oficcinarum).

La fórmula utilizada para determinar la Materia Seca es la siguiente: (PH-PS) / PS $x 100$. 


\section{Rentabilidad del sistema}

Para determinar la rentabilidad del sistema es necesario tener en cuenta los costos de establecimiento de una hectárea de Cratylia argéntea y una hectárea de caña de azúcar (Saccharum Oficcinarum) esto basados en carta tecnológica para cada especie, una vez obtenidos los costos de establecimiento se suman los costos de mantenimiento y cosecha durante 5 años de vida útil para cada especie y se divide entre los mismo 5 años para saber el costo anual.

Una vez determinados los rendimientos promedios de mv/ha/año para ambas especies, se realiza la operación de dividir los rendimientos entre los costos para determinar el valor de un kilogramo de materia verde de cada especie y luego se aplica a la ración suministrada.

Finalmente se estima la producción de leche durante el periodo de estudio y por ende los ingresos económicos que ésta genera. Luego de establecidos tanto los costos de producción como los ingresos obtenidos se define la rentabilidad del sistema. De igual manera se determinaron los costos de producción, en función de alimentación de las vacas testigos para identificar el costo para producir un litro de leche sin suplementación (solo pastoreo).

Luego de determinados los costos de producción se hizo la relación costo-beneficio. Esta se identifica dividiendo los ingresos totales entre los costos de producción para ambos tratamientos.

\section{Procesamiento y análisis estadístico}

El procesamiento de los datos obtenidos en el campo producto de la investigación fueron procesados con el paquete estadístico computarizado denominado SAS (Sistema de Análisis Estadístico) se realizó análisis de varianza y separación de medias a través de la prueba de Tukey esto por ser la más indicada para el tipo de estudio.

Posteriormente se realizó la interpretación de los resultados y se elaboraron los respectivos cuadros y/o gráficos.

\section{Resultados y discusión}

Los resultados que a continuación presentamos fueron obtenidos mediante el Sistema de Análisis Estadísticos (SAS) aplicando el modelo lineal general para el análisis de varianza al que a continuación leeremos ANDEVA y la prueba de separación de medias 
de tukey con un alpha $=0.05$, en éstas medias o promedio que tengan letras iguales son estadísticamente iguales.

\section{PRODUCCION LACTEA}

\section{— SUPLEMENTO - TESTIGOS}

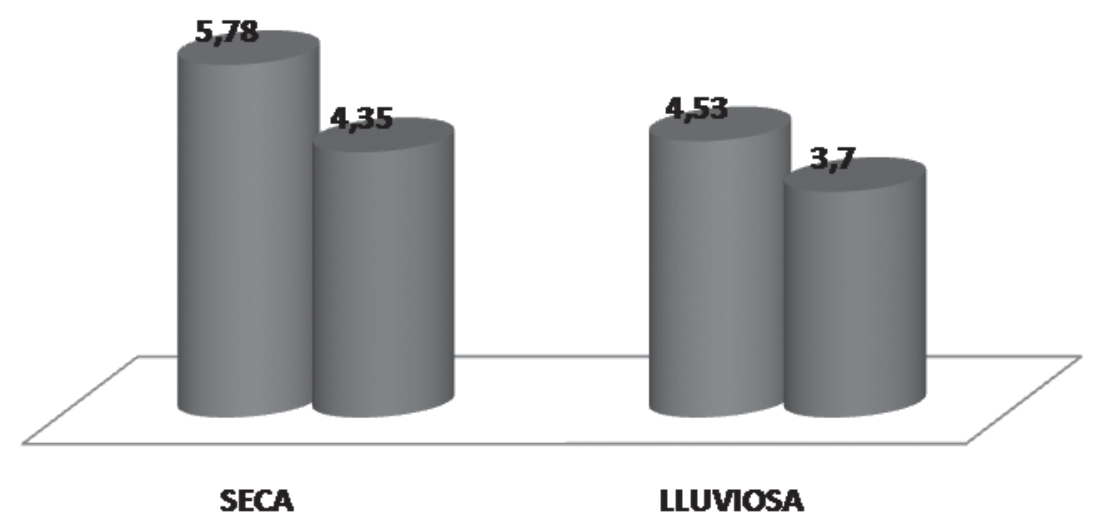

Gráfico 1: Gráfico de varianza para el rendimiento de la producción de la leche bovina (Kg/vaca/día) en ambos tratamientos, durante las dos épocas de año (seca y lluviosa).

\section{Promedios con letras iguales son estadísticamente iguales entre si.}

El gráfico muestra El ANDEVA para la variable producción de leche en vacas suplementadas y sin suplemento en las época seca y lluviosa e indica que los tratamientos son estadísticamente iguales entre sí $(\mathrm{Pr}>0.05)$ prevaleciendo la hipótesis nula con un $95 \%$ de confianza, además del coeficiente de variación (19.52\%) esto demuestra que el experimento fue bien manejado, o sea que posee la capacidad de predecir el alto grado de precisión de estas conclusiones.

Es importante destacar que aunque los promedios de producción de leche por vaca por día son estadísticamente iguales en vacas con y sin suplemento alimenticio, cuantitativamente existe una diferencia de 1.43 correspondiente a la época seca y 0,83 para la época lluviosa, lo que demuestra que existe influencia positiva por producto de la suplementación brindada además de poseer resultados similares a los reportados 
por (INTA, 2005) en estudios de investigación con Cratylia argentea que reportan un incremento en la producción de leche/vaca/día entre 0.75 a 1,5 litros de leche.

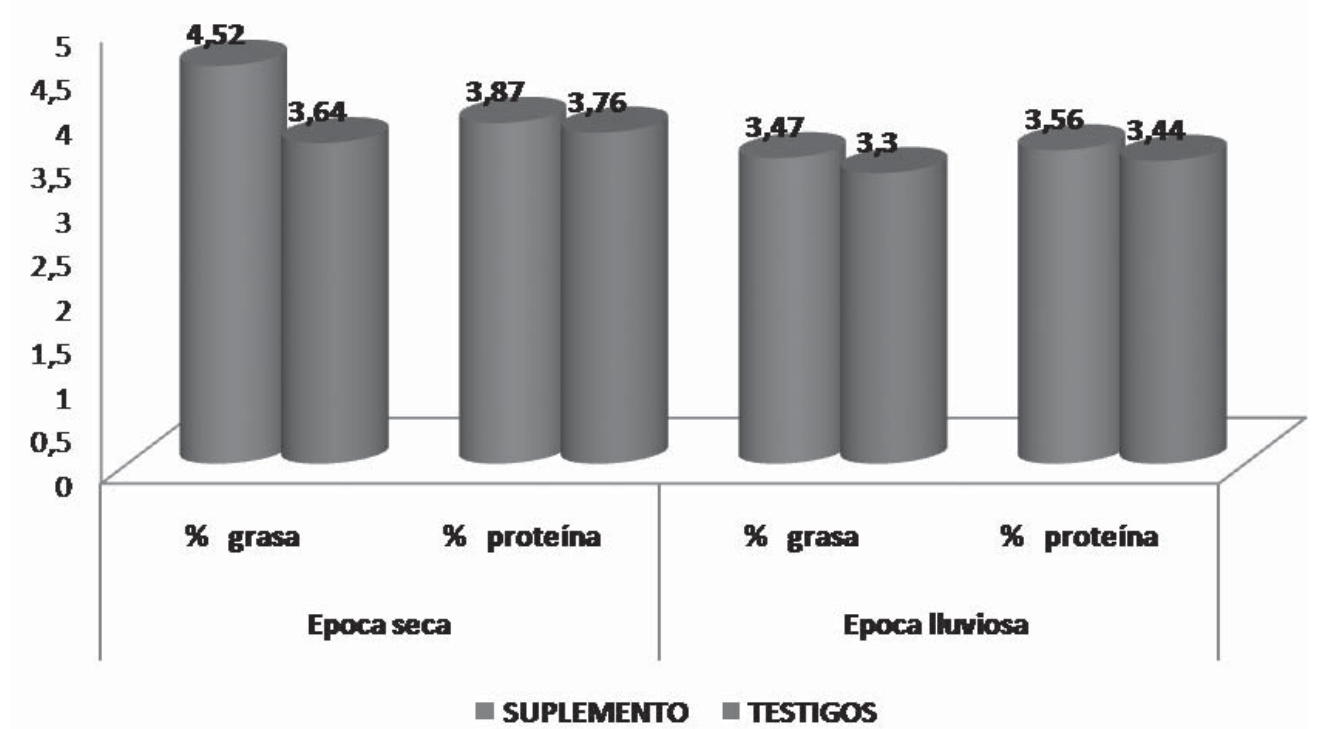

Gráfico 2: Análisis de varianza para el porcentaje de grasa y proteína de la leche en vacas con y sin suplemento alimenticio en la época seca y lluviosa

\section{Promedios con letras iguales son estadísticamente iguales entre sí.}

En el gráfico para la producción de grasa y proteína para la época seca y lluviosa indica que los tratamientos son estadísticamente iguales entre sí, lo que hace prevalecer la hipótesis nula, pero a la vez los valores de coeficiente de variación indican que el estudio fue muy bien manejado lo que le da mayor grado de confiabilidad a los datos obtenidos en nuestra investigación aunque cuantitativamente existe diferencia positiva tanto en grasa como en proteína en ambas épocas en estudio, mostrando mayor diferencia en la época seca con resultados de 0.88 y 0.17 respectivamente.

Por otra parte si analizamos los porcentajes de grasa y proteína para las vacas suplementadas en toda la época de estudio, se obtiene promedios de 4.19 y 3.51 que 
también indica una diferencia cuantitativa con respecto a las vacas testigos de 0.49 $\%$ para grasa y $0.14 \%$ para la proteína.

De acuerdo al Informe anual de la Unidad de Mejoramiento Genético manejado por URACCAN recinto Nueva Guinea, en convenio con el Instituto de Desarrollo Rural (IDR) los porcentajes de grasa y proteína de la leche fueron de 3.83 y 3.37 respectivamente los cuales son similares a los datos generados de las vacas testigos, lo que da mayor veracidad a nuestra investigación.

\section{Producción de materia verde (Mv) y materia seca (Ms) de Cratylia argéntea}

Con relación a la producción de materia verde existe una producción de 15,200kg/ $\mathrm{Mv} / \mathrm{ha} /$ corte, en los cuatro cortes planificados se calculan $60,800 \mathrm{Kg} . / \mathrm{Mv} / \mathrm{Ha}$, que equivale a $60.8 \mathrm{Ton} / \mathrm{Mv} / \mathrm{año}$ como promedio de producción en las dos épocas del año, los que son superiores a los reportados por Miranda, (2004) en Nueva Guinea, RAAS que obtuvo $56.69 \mathrm{Ton} / \mathrm{Mv} / \mathrm{ha}$ con un intervalo de cortes cada 12 semanas equivalente a los cuatro cortes por año.

En cuanto a la materia seca se obtuvieron rendimientos promedios de $0.34 \mathrm{Kg} . /$ $\mathrm{Ms} / \mathrm{m} 2$ lo que equivale $13.92 \mathrm{Ton} / \mathrm{Ms} / \mathrm{Ha}$ con un $22.90 \%$ de Materia seca en la planta similar a los resultados obtenidos de 13.16 tonMs/ha con $23.17 \%$, de materia seca por Miranda (2004).

\section{Producción de materia verde (Mv) y materia seca (Ms) de caña de azúcar (Saccharum officinarum)}

En lo referente a la caña de azúcar (Saccharum officinarum) se encontró un rendimiento de $90.90 \mathrm{Ton} / \mathrm{Mv} / \mathrm{Ha}$ en un corte y un total de 363.6 Ton/Mv/Ha /año en 4 cortes y los rendimientos de $25 \%$ de materia seca en la caña de azúcar dan como resultado 90.90 Ton/Ms/año.

Las vacas durante el estudio de suplementación consumieron 15 Kgs/Mv/día y $300 \mathrm{Kgs}$ de materia verde total por vaca y en materia seca consumieron 3.75 y 75 Kgs/ Ms/vaca respectivamente.

\section{Suministro de Caña de azúcar y Cratylia Argentea}

A las vacas en estudio, individualmente se le suministró $5 \mathrm{Kg}$ de Cratylia argentea en materia verde por día equivalente a $1.14 \mathrm{Kg} / \mathrm{Ms} /$ día y en el estudio de 20 días de Suplementación consumieron $100 \mathrm{Kgs}$ de Mv y 22.9 Kgs de Ms. Y 15 Kg. de caña de 
azúcar (Saccharum officinarum) en materia verde por día equivalente a $3.75 \mathrm{Kg}$. Ms/ día consumiendo $300 \mathrm{Kg}$ de $\mathrm{Mv}$ en todo el estudio y $75 \mathrm{Kg}$ de materia seca.

El suministro total de materia verde en la suplementación (Cratylia argénte) más caña de azúcar (Saccharum officinarum) fue de $20 \mathrm{Kg} / \mathrm{Mv} / \mathrm{vaca} /$ día lo que corresponde al 50\% del consumo diario de una vaca con peso promedio de $400 \mathrm{Kg}$, y de materia seca $4.89 \mathrm{Kgs} / \mathrm{Ms} / \mathrm{vaca} /$ día.

\section{Rentabilidad del sistema}

El presupuesto para el establecimiento de una hectárea de veranera (Cratylia argentea) (ver tabla 14) como fuente proteica indican costos de $C \$ 8,520.45$ éste previendo una vida útil de 5 años. Por lo tanto el costo de un kilo de forraje verde de veranera (Cratylia argentea) tomando en cuenta que se brinda tanto la hoja como el tallo fresco picado es de $C \$ 0.14$, el mismo sirve de base para el análisis económico final y se encontró al dividir el costo del establecimiento entre el rendimiento que fue de 60,800 kilos de $\mathrm{Mv} / \mathrm{Ha} /$ Año.

El costo de una ración de 5 kilos/vaca/día de veranera (Cratylia argentea) en la suplementación realizada fue de $C \$ 0.70$, cada vaca consumió individualmente durante todo el estudio la cantidad de 100 kilos.

Para la caña de azúcar el costo de establecimiento fue de $C \$ 9,692.4$ para un hectárea y los rendimientos fueron de 363,636.36 kilos/año y el costo por cada kilo de caña de azúcar al dividir los costos entre los rendimientos nos arroja un valor de $C \$$ 0.030 / kilo, el costo de la ración de $15 \mathrm{~kg} / \mathrm{vaca} /$ día es de $C \$ 0.45$ cada vaca consumió en todo el estudio 300 kilos de caña de azúcar.

La suplementación tiene un costo de $C \$ 1.15$ /vaca/ día en 20 kilos que se ofreció de los cuales 5 kilos fueron de Cratylia y 15 de caña de azúcar.

Para lograr obtener los resultados de ingresos y egresos se elaboraron 5 cuadros que incluyen costos de establecimiento de ambos cultivos, evaluación de producción y comparación de rentabilidad de vacas suplementadas y testigos las que pueden apreciarse en anexos. 


\section{SISTEMA AGROFORESTAL Y PRODUCCIÓN PECUARIA}

CUADRO 5: Comparación de la rentabilidad en vacas suplementadas y sin suplemento Testigo.

\begin{tabular}{|c|c|c|c|c|c|}
\hline \multirow{2}{*}{ No. } & \multirow{2}{*}{ Descripción } & \multirow{2}{*}{ Suplementadas } & \multirow{2}{*}{ Testigos } & \multicolumn{2}{|c|}{ Diferencia } \\
\hline & & & & Cs & Us\$ \\
\hline 1 & $\begin{array}{l}\text { Total de ingresos leche de } 5 \\
\text { vacas }\end{array}$ & $37.563,75$ & $29.767,50$ & 7796,25 & 423,71 \\
\hline 2 & Total Egresos & $8.330,50$ & 10180 & $-1849,5$ & $-100,52$ \\
\hline 3 & Utilidad Total & $29.233,25$ & $19.587,50$ & 9645,75 & 524,23 \\
\hline 4 & Utilidad /Vaca/Lactancia & $5.846,65$ & $3.917,50$ & 1929,15 & 104,85 \\
\hline 5 & Utilidad/mes lactancia/vaca & 649,63 & 435,28 & 214,35 & 11,65 \\
\hline 6 & Utilidad/5 vacas/dìa. & 108,27 & 72,55 & 35,73 & 1,94 \\
\hline 7 & Utilidad /vaca/dìa & 21,65 & 14,51 & 7,15 & 0,39 \\
\hline 8 & Utilidad adicional del ternero & 12,96 & 11,85 & 1,11 & 0,06 \\
\hline 9 & $\begin{array}{l}\text { Utilidad total/lactancia/vaca/ } \\
\text { dìa (leche +ter nero) }\end{array}$ & 34,60 & 26,36 & 8,26 & 0,45 \\
\hline 10 & Relación beneficio costo & $1: 4.5$ & $1: 2.92$ & & \\
\hline 11 & Rentabilidad del sistema \% & 350 & 192 & & \\
\hline
\end{tabular}

El cuadro $\mathrm{N}^{\circ} 5$ muestra el análisis comparativo entre vacas suplementadas y testigos se puede apreciar que la producción de leche en los 9 meses en vacas suplementadas generó una utilidad adicional de $C \$ 9,645.75$ córdobas netos. La utilidad por vaca por lactancia es de $C \$ 1929.15$, al llevar la Suplementación a todo el hato que está compuesto de 70 vacas en producción de leche, de la Finca Rancho bonanza es de esperarse resultados extras de $C \$ 135,040.50$ en una lactancias de 9 meses.

La utilidad adicional/vaca/día fue de C $\$ 7.15$ solamente en lo que a producción de leche se refiere, ahora tomando en cuenta la utilidad adicional que generaría un mejor estado corporal en los terneros de $C \$ 1.11$ al momento del destete, sumaria C\$ 8.26 vaca/día. Si lo aplicamos a las 70 vacas del hato en estudio los resultados de producción de leche más el ternero en 270 días de lactancia resultaría la cantidad total de $C \$ 156,114.00$ de ingresos adicionales producto de la intervención técnica. 


\section{Conclusiones}

El cultivo de veranera (Cratylia argentea) es una alternativa de suplementación nutricional proteica para bovinos mejorando los rendimientos y la calidad de la producción láctea.

Los rendimientos de materia verde y seca resultantes en las especies evaluadas son aceptables, alcanzando producción promedio de $60,800 \mathrm{Kg} / \mathrm{año}$ en veranera (Cratylia argentea) y 363,636.36 Kg en caña de azúcar (Saccharum offcinarum) suficiente para suplir los requerimientos proteicos y energéticos del ganado bovino en producción láctea.

La ración ofrecida durante la investigación nos brindó resultados económicos satisfactorios al incrementarse la producción/vaca/día, mejorando la rentabilidad del sistema, además los costos de la ración a base de las dos plantas mencionadas es barata y sostenible.

La suplementación brindada en la época seca presentó mejores resultados en función del aumento de la producción y calidad de la leche, esto porque la ración ofrecida fue aprovechada en su totalidad.

La relación beneficio costo de la suplementación alimenticia es de 1/4.50 lo que significa que por cada córdoba invertido se generan 3.50 córdobas de utilidad. Para una rentabilidad del $350 \%$.

\section{Lista de referencias}

Argel, P.J.; Lascano, C.E. 1995. Cratylia argentea: Una Nueva Leguminosa Arbustiva para Suelos Ácidos en Zonas Subhúmedas Tropicales.

CENAGRO (2002). Tercer Censo Nacional Agropecuario. INEC (Instituto Nacional de Estadísticas y Censos).

CIAT (1997) La Cratylia argentea: una nueva leguminosa arbustiva para suelos ácidos en zonas sub húmedas tropicales. Cali, Colombia;.

CIAT. (1991). Semillas de especies forrajeras tropicales, programa para desarrollo de capacitación científica en investigación para producción y utilización de pastos. Cali - Colombia. 


\section{SISTEMA AGROFORESTAL Y PRODUCCIÓN PECUARIA}

Fundación Hogares Juveniles Campesinos . (2002). Manual Agropecuario. Bogotá.

INTA. (2005). Suplementación de vacas lecheras. Centro Norte, Nicaragua .

Maass, B. L. (1995). Evaluación Agronómica de Cratylia argentea (desvaux) O. Kuntze en Colombia. Potencial del genero Cratylia Como leguminosa forrajera.

Maass, B.L.; SCHULTZE-KRAFT, R. ; ARGEL, P. s.f. Revisión de la evaluación agronómica de especies arbustivas. En: Pizarro, E.A. y Coradin, L. (eds). Potencial del género Cratylia como leguminosa forrajera. Memorias del taller de trabajo sobre Cratylia realizado el 19 y 20 de julio de 1995. Brasilia, Brasil. 117 p. p. 107-114.

Miranda B, JC (2004). Producción de biomasa de la leguminosa arbustiva Cratylia argentea bajo el efecto de diferentes densidades de siembra y frecuencias de corte en el municipio de Nueva Guinea, Nicaragua.

Pizarro, E. A. (1995). Introducción y evaluación de leguminosas forrajeras arbustivas en el cerrado brasileño. P. 40-49.

PRA-DC/IDR (2005). Informe Técnico Anual Cuadrilátero Lechero, Programa de Rehabilitación Arrocera. Instituto de Desarrollo Rural, Cooperación Italiana (PRA-DC/IDR). Nueva Guinea, RAAS. Nicaragua.

Página de información ganadera de ray del pino (2000). Geocities.Recuperado el 2007, de http://www.geocities.com

Urdaneta, J. (2005). AVPA (Asociación Venezolana de producción ganadera). Recuperado el 2007, de htpp//www.geocities.com 